\title{
A descriptive, additive modification of Mawhin's integral and the Divergence Theorem with singularities
}

\author{
by Dirk Jens F. Nonnenmacher (Ulm)
}

\begin{abstract}
Modifying Mawhin's definition of the $G P$-integral we define a well-behaved integral over $n$-dimensional compact intervals. While its starting definition is of Riemann type, we also establish an equivalent descriptive definition involving characteristic null conditions. This characterization is then used to obtain a quite general form of the divergence theorem.

0. Introduction. It is well known that, in contrast to the one-dimensional situation, the Denjoy-Perron integral in higher dimensions does not integrate the divergence of any differentiable vector field. To remove this deficiency Mawhin [Maw] introduced the $G P$-integral (over intervals) which indeed yields the divergence theorem without any integrability assumptions. Although the integral has further satisfactory properties its main lack is the complete failure of additivity, i.e. if an interval is subdivided into two intervals on each of which a function is integrable, the function need not be integrable on the original interval. In the meanwhile different additive integration processes, preserving the good properties of Mawhin's integral, have been presented in $[\mathrm{JKS}],[\mathrm{Pf}],[\mathrm{Ju}-\mathrm{No}]$.

All integrals mentioned so far are of Riemann type and their definitions involve $\delta$-fine partitions which are further restricted by different means. While Mawhin only uses partitions in which all intervals satisfy a (Vitali) regularity condition, Pfeffer $[\mathrm{Pf}]$ employs a relative regularity with respect to a finite family of $k$-planes parallel to the coordinate axes. In contrast to these local restrictions a global one, not dealing with regularity of single intervals, is used in $[\mathrm{JKS}]$. Here a certain expression which is associated with the partition is required to be bounded.
\end{abstract}

1991 Mathematics Subject Classification: 26A39, 26B20.

Key words and phrases: Mawhin's integral, additive interval function, divergence theorem. 
In this paper we define a well-behaved integral over $n$-dimensional compact intervals employing a new and simple type of restriction: we consider only those $\delta$-fine partitions for which the sum of the $(n-1)$-dimensional measures of the boundaries of the non-regular intervals occurring in the partition is bounded. Thus our restriction has a global character but maintains the original concept of Vitali regularity. It will be shown that our integral extends the one of $[\mathrm{JKS}]$ but is more restrictive than the integrals discussed in $[\mathrm{Pf}]$ and $[\mathrm{Ju}-\mathrm{No}]$.

After having established the basic properties of our integral (as linearity and additivity) we will introduce two types of null conditions for an interval function. As a consequence we will be able to characterize our integral as an additive, almost everywhere differentiable interval function satisfying these null conditions. In particular, we see that an interval function is an indefinite integral in our sense iff it is an indefinite $G P$-integral and satisfies a further null condition. The latter thus guarantees the additivity.

This descriptive definition of our integral is then used to show that our process of integration extends the one of Lebesgue, and to establish a quite general divergence theorem. In particular, the vector field may not be differentiable on countably many hyperplanes and its continuity may fail at countably many singularities where we only assume the vector field to be of Lipschitz type with a non-positive exponent $\alpha>1-n$. This result generalizes the ones in [Maw], [JKS] but is contained in the ones of $[\mathrm{Pf}]$ and $[\mathrm{Ju}-\mathrm{No}]$. For an "optimal" version of the divergence theorem (generalizing in particular the result in $[\mathrm{Pf}]$ ) the reader is referred to [Ju-No].

1. Preliminaries. We denote by $\mathbb{R}$ and $\mathbb{R}^{+}$the set of all real and all positive real numbers, respectively. Throughout the paper $n$ is a fixed positive integer and we work in $\mathbb{R}^{n}$ with the usual inner product $x \cdot y=\sum_{i=1}^{n} x_{i} y_{i}$ $\left(x=\left(x_{i}\right), y=\left(y_{i}\right) \in \mathbb{R}^{n}\right)$ and the associated norm $\|\cdot\|$. For $x \in \mathbb{R}^{n}$ and $r>0$ we let $B(x, r)=\left\{y \in \mathbb{R}^{n}:\|y-x\| \leq r\right\}$.

If $E \subseteq \mathbb{R}^{n}$ we denote by $E^{\circ}, \partial E, d(E)$ and $|E|_{n}$ the interior, boundary, diameter and the outer Lebesgue measure of $E$, respectively. Terms like measurable and almost everywhere (a.e.) always refer to the Lebesgue measure $|\cdot|_{n}$, and $E \subseteq \mathbb{R}^{n}$ is called an $n$-null set if $|E|_{n}=0$. The distance of a point $x \in \mathbb{R}^{n}$ to a set $E \subseteq \mathbb{R}^{n}$ is denoted by $\operatorname{dist}(x, E)$.

By $|\cdot|_{n-1}$ we denote the $(n-1)$-dimensional normalized outer Hausdorff measure in $\mathbb{R}^{n}$ which coincides on $\mathbb{R}^{n-1}\left(\subseteq \mathbb{R}^{n}\right)$ with the $(n-1)$-dimensional outer Lebesgue measure $\left(|\cdot|_{0}\right.$ being the counting measure). Instead of $|\cdot|_{n-1}$ we also write $\mathcal{H}(\cdot)$.

An interval $I$ in $\mathbb{R}^{n}$ is always assumed to be compact and non-degenerate and its regularity $r(I)$ is defined as the ratio of the smallest and the largest 
edges of $I$. Finitely many intervals are said to be non-overlapping if they have pairwise disjoint interiors.

By a plane (in $\mathbb{R}^{n}$ ) we always mean an $(n-1)$-dimensional linear submanifold of $\mathbb{R}^{n}$ which is parallel to $n-1$ distinct coordinate axes.

2. The integral and its basic properties. In this section we give the definition of our integral, we establish its relation to other integrals and we prove its basic properties such as additivity.

Given an interval $I$, an interval function $F$ on $I$ associates with any subinterval $J$ of $I$ a real number $F(J)$. We call $F$ additive if $F(J)=\sum F\left(J_{k}\right)$ for any subinterval $J$ of $I$ and any finite sequence of non-overlapping intervals $J_{k}$ whose union is $J$.

If $x \in I^{\circ}$ we call an interval function $F$ on $I$ differentiable at $x$ if it is derivable in the ordinary sense at $x$ according to [Saks], and in that case $F^{\prime}(x)$ denotes the ordinary derivative of $F$ at $x$.

Let $E \subseteq \mathbb{R}^{n}$ and $\delta: E \rightarrow \mathbb{R}^{+}$be given. Then a finite sequence of pairs $\left\{\left(x_{i}, I_{i}\right)\right\}$ is called $(E, \delta)$-fine if the $I_{i}$ are non-overlapping intervals, $x_{i} \in E \cap I_{i}$ and $d\left(I_{i}\right)<\delta\left(x_{i}\right)$ for all $i$. If furthermore $E=\bigcup I_{i}$ we call $\left\{\left(x_{i}, I_{i}\right)\right\}$ a $\delta$-fine partition of $E$.

Given an interval $I, r>0, K \geq 0$ and $\delta: I \rightarrow \mathbb{R}^{+}$we denote by $\mathcal{P}(I, r, K, \delta)$ the collection of all $\delta$-fine partitions $\Pi=\left\{\left(x_{i}, I_{i}\right)\right\}$ of $I$ satisfying $\sum_{r\left(I_{i}\right)<r}\left|\partial I_{i}\right|_{n-1} \leq K$.

If $f$ denotes a real-valued function defined on $I$ we write $S(f, \Pi, I)=$ $\sum f\left(x_{i}\right)\left|I_{i}\right|_{n}$ for any $\delta$-fine partition $\Pi=\left\{\left(x_{i}, I_{i}\right)\right\}$ of $I$.

Definition 2.1. Let $I$ be an interval and $f: I \rightarrow \mathbb{R}$ be a function. Then we call $f$ integrable on $I$ if there exists a real number $\gamma$ with the property that for any $\varepsilon>0, r>0, K>0$ there exists a $\delta: I \rightarrow \mathbb{R}^{+}$such that

$$
|\gamma-S(f, \Pi, I)| \leq \varepsilon \quad \forall \Pi \in \mathcal{P}(I, r, K, \delta) .
$$

Remark 2.1. (i) Assume $0<r<1$. Then it is well known that any interval $I$ can be decomposed into finitely many non-overlapping intervals each of which has a regularity $\geq r$. Thus, given in addition a function $\delta: I \rightarrow$ $\mathbb{R}^{+}$, a usual compactness argument yields the existence of a $\delta$-fine partition $\Pi=\left\{\left(x_{i}, I_{i}\right)\right\}$ of $I$ with $r\left(I_{i}\right) \geq r$ for all $i$. Consequently, our definition above is meaningful and $\gamma$ is, if it exists, uniquely determined; we write $\gamma=\int_{I} f$.

(ii) Let $f: I \rightarrow \mathbb{R}$ be $M_{1}$-integrable on $I$ (cf. [JKS]). Then $f$ is integrable on $I$ and both integrals coincide. To see this let $r>0, K>0$ and $\delta: I \rightarrow \mathbb{R}^{+}$ with $\delta(\cdot) \leq 1$ be given, take $\Pi=\left\{\left(x_{i}, I_{i}\right)\right\} \in \mathcal{P}(I, r, K, \delta)$ and observe that 


$$
\begin{aligned}
\sum d\left(I_{i}\right)\left|\partial I_{i}\right|_{n-1} & \leq \sum_{r\left(I_{i}\right)<r}\left|\partial I_{i}\right|_{n-1}+2 n \sum_{r\left(I_{i}\right) \geq r} d\left(I_{i}\right)^{n} \\
& \leq K+2 n\left(\frac{\sqrt{n}}{r}\right)^{n} \sum\left|I_{i}\right|_{n} \leq K+2 n\left(\frac{\sqrt{n}}{r}\right)^{n}|I|_{n} .
\end{aligned}
$$

(iii) Assume $f: I \rightarrow \mathbb{R}$ to be integrable on $I$. Then $f$ is integrable in the sense of $[\mathrm{Pf}]$ and both integrals coincide: let a regulator $(\varepsilon, \mathcal{H})$ and $\delta: I \rightarrow \mathbb{R}^{+}$with $\delta(\cdot) \leq 1$ be given. Then (cf. [No]) it is easy to see that any $\delta$-fine partition $\Pi=\left\{\left(x_{i}, I_{i}\right)\right\}$ of $I$ with $r\left(I_{i}, \mathcal{H}\right) \geq \varepsilon$ for all $i$ satisfies $\sum_{r\left(I_{i}\right)<\varepsilon}\left|\partial I_{i}\right|_{n-1} \leq K=K(\varepsilon, \mathcal{H})$.

(iv) If $f: I \rightarrow \mathbb{R}$ is integrable on $I$, then $f$ is $\nu_{1}$-integrable on $I$ (cf. $[\mathrm{Ju}-\mathrm{No}])$ and both integrals coincide. To see this take $I^{\circ},\left(\partial I, C_{1}^{n-1}\right)$ as a division of $I$ and apply [Ju-No, Thm. 3.1].

Proposition 2.1. If I is an interval, then the family of all integrable functions on $I$ is a linear space, and the map $f \mapsto \int_{I} f$ is a non-negative linear functional on this space.

P r o of. Direct consequence of Definition 2.1.

Proposition 2.2. Let $I$ be an interval, and $f: I \rightarrow \mathbb{R}$.

(i) $f$ is integrable on I iff for every $\varepsilon>0, r>0, K>0$ there is a $\delta: I \rightarrow \mathbb{R}^{+}$such that $\left|S\left(f, \Pi_{1}, I\right)-S\left(f, \Pi_{2}, I\right)\right| \leq \varepsilon$ for all $\Pi_{1,2} \in \mathcal{P}(I, r, K, \delta)$.

(ii) If $f$ is integrable on $I$ then $f$ is integrable on any subinterval of $I$.

Proof. (i) Choose sequences $\varepsilon_{n}>0,1 / 2 \geq r_{n}>0, K_{n}>0$ such that $\varepsilon_{n} \searrow 0, r_{n} \searrow 0, K_{n} \nearrow \infty$ and determine $\delta_{n}: I \rightarrow \mathbb{R}^{+}$according to the Cauchy condition. We may assume $\delta_{n+1}(\cdot) \leq \delta_{n}(\cdot)$ for all $n$, and we choose a $\Pi_{n} \in \mathcal{P}\left(I, 1 / 2,0, \delta_{n}\right)\left(\subseteq \mathcal{P}\left(I, r_{n}, K_{n}, \delta_{n}\right)\right)$ (cf. Remark 2.1(i)). Obviously $S_{n}=S\left(f, \Pi_{n}, I\right)$ is a Cauchy sequence and thus there is a $\gamma \in \mathbb{R}$ with $S_{n} \rightarrow \gamma$.

Now let $\varepsilon>0, r>0, K>0$ be given and determine $n_{0} \in \mathbb{N}$ such that $\varepsilon_{n_{0}} \leq \varepsilon / 2, r_{n_{0}} \leq r, K_{n_{0}} \geq K$ and $\left|\gamma-S_{n_{0}}\right| \leq \varepsilon / 2$. Set $\delta=\delta_{n_{0}}$ and let $\Pi \in \mathcal{P}(I, r, K, \delta) \subseteq \mathcal{P}\left(I, r_{n_{0}}, K_{n_{0}}, \delta_{n_{0}}\right)$. Hence,

$$
|\gamma-S(f, \Pi, I)| \leq\left|\gamma-S_{n_{0}}\right|+\left|S_{n_{0}}-S(f, \Pi, I)\right| \leq \varepsilon / 2+\varepsilon_{n_{0}} \leq \varepsilon .
$$

The other direction is obvious.

(ii) Let $J$ be a subinterval of $I$ and choose finitely many intervals $I_{i}$ such that $I$ is the non-overlapping union of all the intervals $I_{i}$ and $J$. Assume $\varepsilon>0, r>0, K>0$ to be given and determine $\delta: I \rightarrow \mathbb{R}^{+}$according to (i) for $\varepsilon, r^{*}=\min (r, 1 / 2), K$. Take $\Pi^{1,2} \in \mathcal{P}(J, r, K, \delta)$ and choose $\Pi(i) \in \mathcal{P}\left(I_{i}, r^{*}, 0, \delta\right)$ (cf. Remark 2.1(i)). Obviously $\Pi_{1,2}=\Pi^{1,2} \cup \bigcup_{i} \Pi(i) \in$ $\mathcal{P}\left(I, r^{*}, K, \delta\right)$ and consequently

$$
\left|S\left(f, \Pi^{1}, J\right)-S\left(f, \Pi^{2}, J\right)\right|=\left|S\left(f, \Pi_{1}, I\right)-S\left(f, \Pi_{2}, I\right)\right| \leq \varepsilon .
$$


Our next aim is to prove the complete additivity of our integral. We first establish the following simple but useful lemma.

Lemma 2.1. Let $r>0$ and a plane $H$ be given. Then

$$
|\partial J|_{n-1} \leq 2 n|H \cap J|_{n-1} / r
$$

for any interval $J$ with $H \cap J \neq \emptyset$ and $r(J) \geq r$.

Proof. Without loss of generality assume $H$ to be perpendicular to the $x_{1}$-axis. Then, denoting by $l_{j}$ the lengths of the sides of $J$, we have $|H \cap J|_{n-1}=\prod_{j \neq 1} l_{j}$, and by regularity $l_{1} \leq l_{i} / r$ for all $i$. Hence,

$$
\begin{aligned}
|\partial J|_{n-1} & =2 \sum_{i=1}^{n} \prod_{\substack{j=1 \\
j \neq i}}^{n} l_{j}=2\left(|H \cap J|_{n-1}+\sum_{i=2}^{n} l_{1} \prod_{\substack{j=2 \\
j \neq i}}^{n} l_{j}\right) \\
& \leq 2 n|H \cap J|_{n-1} / r \quad \text { since } r \leq 1 .
\end{aligned}
$$

Proposition 2.3. Let the interval $I$ be the non-overlapping union of intervals $I_{1}$ and $I_{2}$, and assume the function $f: I \rightarrow \mathbb{R}$ to be integrable on $I_{1}$ and $I_{2}$. Then $f$ is integrable on $I$ and $\int_{I} f=\int_{I_{1}} f+\int_{I_{2}} f$.

P r o of. We denote by $H$ the plane determined by $H \cap I=I_{1} \cap I_{2}$. Assume $\varepsilon>0, r>0, K>0$ to be given, and for $\varepsilon / 2, r, K^{*}=K+2^{n+1} n|H \cap I|_{n-1} / r$ determine $\delta_{1,2}: I_{1,2} \rightarrow \mathbb{R}^{+}$such that $\left|S\left(f, \Pi_{1,2}, I_{1,2}\right)-\int_{I_{1,2}} f\right| \leq \varepsilon / 2$ for all $\Pi_{1,2} \in \mathcal{P}\left(I_{1,2}, r, K^{*}, \delta_{1,2}\right)$. We define $\delta: I \rightarrow \mathbb{R}^{+}$by

$$
\delta(x)= \begin{cases}\min \left(\delta_{1}(x), \operatorname{dist}\left(x, I_{2}\right)\right), & x \in I_{1}-I_{2}, \\ \min \left(\delta_{2}(x), \operatorname{dist}\left(x, I_{1}\right)\right), & x \in I_{2}-I_{1}, \\ \min \left(\delta_{1}(x), \delta_{2}(x)\right), & x \in I_{1} \cap I_{2} .\end{cases}
$$

Let $\Pi=\left\{\left(x_{i}, I_{i}\right)\right\} \in \mathcal{P}(I, r, K, \delta)$ and set $\Pi_{1,2}=\left\{\left(x_{i}, I_{i} \cap I_{1,2}\right):\left(x_{i}, I_{i}\right) \in\right.$ $\Pi\}$ (avoiding degenerations). We will show that $\Pi_{1,2} \in \mathcal{P}\left(I_{1,2}, r, K^{*}, \delta_{1,2}\right)$ and then

$$
\begin{aligned}
& \left|S(f, \Pi, I)-\left(\int_{I_{1}} f+\int_{I_{2}} f\right)\right| \\
& \quad \leq\left|S\left(f, \Pi_{1}, I_{1}\right)-\int_{I_{1}} f\right|+\left|S\left(f, \Pi_{2}, I_{2}\right)-\int_{I_{2}} f\right| \leq \varepsilon
\end{aligned}
$$

as desired.

Obviously $\Pi_{1}$ is a $\delta_{1}$-fine partition of $I_{1}$. Furthermore,

$$
\begin{aligned}
\sum_{r\left(I_{i} \cap I_{1}\right)<r}\left|\partial\left(I_{i} \cap I_{1}\right)\right|_{n-1} & \leq \sum_{r\left(I_{i}\right)<r, x_{i} \in I_{1}-I_{2}}\left|\partial I_{i}\right|_{n-1}+\sum_{x_{i} \in H}\left|\partial I_{i}\right|_{n-1} \\
& \leq \sum_{r\left(I_{i}\right)<r}\left|\partial I_{i}\right|_{n-1}+\sum_{r\left(I_{i}\right) \geq r, x_{i} \in H}\left|\partial I_{i}\right|_{n-1}
\end{aligned}
$$




$$
\begin{aligned}
& \leq K+\frac{2 n}{r} \sum_{x_{i} \in H}\left|H \cap I_{i}\right|_{n-1} \\
& \leq K^{*} \quad \text { by the above lemma. }
\end{aligned}
$$

Remark 2.2. Let $I$ be an interval and $f: I \rightarrow \mathbb{R}$ be integrable. Then we can define an additive interval function $F$ on $I$ by $F(J)=\int_{J} f$ for each subinterval $J$ of $I$. We call $F$ the indefinite integral of $f$.

3. A descriptive definition of the integral. Our next aim is to give a characterization of the integral in terms of an additive, a.e. differentiable interval function satisfying two further null conditions.

We first prove our version of the Saks-Henstock Lemma.

Lemma 3.1. Suppose $I$ is an interval and let $f: I \rightarrow \mathbb{R}$ be a function. Then $f$ is integrable on $I$ iff there exists an additive interval function $F$ on $I$ having the following property:

$$
\begin{aligned}
\forall \varepsilon>0, r>0, K>0 & \exists \delta: I \rightarrow \mathbb{R}^{+} \text {such that } \\
& \left.\sum\left|F\left(I_{i}\right)-f\left(x_{i}\right)\right| I_{i}\right|_{n} \mid \leq \varepsilon
\end{aligned}
$$

for any $(I, \delta)$-fine sequence $\left\{\left(x_{i}, I_{i}\right)\right\}$ satisfying $I_{i} \subseteq I$ for all $i$ and $\sum_{r\left(I_{i}\right)<r}\left|\partial I_{i}\right|_{n-1} \leq K$. In any case we have $\int_{I} f=F(I)$.

Pro of. Since one part of the assertion is obvious, we assume $f$ to be integrable. Denote by $F$ the indefinite integral of $f$, suppose positive numbers $\varepsilon, r, K$ to be given and let $r^{*}=\min (r, 1 / 2)$. By the integrability of $f$ we can find a $\delta: I \rightarrow \mathbb{R}^{+}$such that

$$
|F(I)-S(f, \Pi, I)| \leq \varepsilon / 4 \quad \forall \Pi \in \mathcal{P}\left(I, r^{*}, K, \delta\right),
$$

and we denote by $\left\{\left(x_{i}, I_{i}\right): 1 \leq i \leq m\right\}$ a (non-empty) $(I, \delta)$-fine sequence with $I_{i} \subseteq I$ and $\sum_{r\left(I_{i}\right)<r}\left|\partial I_{i}\right|_{n-1} \leq K$. By rearranging (if necessary) we determine $\mu$ with $0 \leq \mu \leq m$ such that $F\left(I_{i}\right) \geq f\left(x_{i}\right)\left|I_{i}\right|_{n}$ for $1 \leq i \leq \mu$ and $F\left(I_{i}\right)<f\left(x_{i}\right)\left|I_{i}\right|_{n}$ if $\mu+1 \leq i \leq m$. Furthermore, we choose (if possible) intervals $J_{1}, \ldots, J_{p}\left(p \in \mathbb{N}_{0}\right)$ such that $I$ is the non-overlapping union of all the $I_{i}, J_{k}$, and we set $\alpha=m+p$.

For any subinterval $J$ of $I$ we may choose a $\delta_{J}: J \rightarrow \mathbb{R}^{+}$with $\delta_{J}(\cdot) \leq \delta(\cdot)$ on $J$ such that

$$
|F(J)-S(f, \Pi(J), J)| \leq \varepsilon /(4 \alpha) \quad \forall \Pi(J) \in \mathcal{P}\left(J, r^{*}, K, \delta_{J}\right),
$$

and we fix a $\Pi(J) \in \mathcal{P}\left(J, r^{*}, 0, \delta_{J}\right)$ (cf. Remark 2.1(i)). Now it is immediate that

$$
\Pi=\left\{\left(x_{i}, I_{i}\right): 1 \leq i \leq \mu\right\} \cup \bigcup_{i=\mu+1}^{m} \Pi\left(I_{i}\right) \cup \bigcup_{k=1}^{p} \Pi\left(J_{k}\right) \in \mathcal{P}\left(I, r^{*}, K, \delta\right),
$$


which yields

$$
\begin{aligned}
\varepsilon / 4 \geq & F(I)-S(f, \Pi, I) \\
= & \left.\sum_{i=1}^{\mu}\left|F\left(I_{i}\right)-f\left(x_{i}\right)\right| I_{i}\right|_{n} \mid+\sum_{i=\mu+1}^{m}\left[F\left(I_{i}\right)-S\left(f, \Pi\left(I_{i}\right), I_{i}\right)\right] \\
& +\sum_{k=1}^{p}\left[F\left(J_{k}\right)-S\left(f, \Pi\left(J_{k}\right), J_{k}\right)\right] .
\end{aligned}
$$

Consequently, $\left.\sum_{i=1}^{\mu}\left|F\left(I_{i}\right)-f\left(x_{i}\right)\right| I_{i}\right|_{n} \mid \leq \varepsilon / 2$, and the remaining inequality $\left.\sum_{i=\mu+1}^{m}\left|F\left(I_{i}\right)-f\left(x_{i}\right)\right| I_{i}\right|_{n} \mid \leq \varepsilon / 2$ follows analogously.

Next we use Lemma 3.1 to prove that the indefinite integral $F$ of an integrable function $f$ is differentiable a.e. with $F^{\prime}=f$.

Lemma 3.2. Let $f: I \rightarrow \mathbb{R}$ be integrable on the interval $I$ and denote by $F$ the indefinite integral of $f$. Then $F$ is differentiable a.e. on I with $F^{\prime}=f$.

Proof. We denote by $E$ the set of all $x \in I^{\circ}$ at which $F$ is not differentiable or where $F^{\prime}(x) \neq f(x)$; we have to show $|E|_{n}=0$. Clearly for each $x \in E$ we can find positive numbers $\varepsilon(x)$ and $r(x)$ such that for any $\delta>0$ there exists a subinterval $J$ of $I$ with $x \in J, d(J)<\delta, r(J) \geq r(x)$ and $\left.\left.|F(J)-f(x)| J\right|_{n}|>\varepsilon(x)| J\right|_{n}$.

For $m \in \mathbb{N}$ we let $E_{m}=\{x \in E: \varepsilon(x) \geq 1 / m, r(x) \geq 1 / m\}$ and it suffices to prove $\left|E_{m}\right|_{n}=0$. Thus let $\varepsilon>0$ and choose a $\delta: I \rightarrow \mathbb{R}^{+}$ according to Lemma 3.1 for the parameters $\varepsilon / m, r=1 / m, K=1$. We denote by $V$ the family of all subintervals $J$ of $I$ with $r(J) \geq 1 / m$ for which there exists an $x \in J \cap E_{m}$ such that $d(J)<\delta(x)$ and $\left.|F(J)-f(x)| J\right|_{n} \mid \geq$ $|J|_{n} / m$. A glance shows that $V$ is a Vitali cover of $E_{m}$, and thus there are (at most) countably many disjoint intervals $J_{i} \in V$ with $\left|E_{m}-\bigcup_{i} J_{i}\right|_{n}=0$. To each $J_{i}$ there corresponds by definition a point $x_{i} \in J_{i} \cap E_{m}$ and since for each $p \in \mathbb{N}$ the sequence $\left\{\left(x_{i}, J_{i}\right): 1 \leq i \leq p\right\}$ satisfies the requirements of Lemma 3.1 we get

$$
\left|E_{m}\right|_{n} \leq \lim _{p \rightarrow \infty} \sum_{i=1}^{p}\left|J_{i}\right|_{n} \leq\left.\limsup _{p \rightarrow \infty} m \sum_{i=1}^{p}\left|F\left(J_{i}\right)-f\left(x_{i}\right)\right| J_{i}\right|_{n} \mid \leq \varepsilon,
$$

hence $\left|E_{m}\right|_{n}=0$.

R e mark 3.1. In the sequel we will need the following simple facts.

(i) Assume an $n$-null set $E$ and a function $f: E \rightarrow \mathbb{R}$ to be given. Then for any $\varepsilon>0$ there exists a $\delta: E \rightarrow \mathbb{R}^{+}$such that $\sum\left|f\left(x_{i}\right) \| I_{i}\right|_{n} \leq \varepsilon$ for any $(E, \delta)$-fine sequence $\left\{\left(x_{i}, I_{i}\right)\right\}$. For, let $E_{k}=\{x \in E: k-1 \leq$ $|f(x)|<k\} \quad(k \in \mathbb{N})$, find open sets $G_{k} \supseteq E_{k}$ with $\left|G_{k}\right|_{n} \leq \varepsilon /\left(k 2^{k}\right)$ and 
for $x \in E_{k}$ choose a $\delta(x)>0$ such that $B(x, \delta(x)) \subseteq G_{k}$. Then for any $(E, \delta)$-fine sequence $\left\{\left(x_{i}, I_{i}\right)\right\}$ we get

$$
\sum\left|f\left(x_{i}\right)\right|\left|I_{i}\right|_{n} \leq \sum_{k} k \sum_{x_{i} \in E_{k}}\left|I_{i}\right|_{n} \leq \sum_{k} k\left|G_{k}\right|_{n} \leq \varepsilon,
$$

as desired.

(ii) Denote by $I$ an interval and let $f: I \rightarrow \mathbb{R}$ be a function. Then for any $\varepsilon>0, K>0$ there is a $\delta: I \rightarrow \mathbb{R}^{+}$such that $\sum\left|f\left(x_{i}\right)\right|\left|I_{i}\right|_{n} \leq \varepsilon$ for any $(I, \delta)$-fine sequence $\left\{\left(x_{i}, I_{i}\right)\right\}$ satisfying $\sum\left|\partial I_{i}\right|_{n-1} \leq K$. For, let $\varepsilon>0, K>0$ be given and set $\delta(\cdot)=\varepsilon /(K(1+|f(\cdot)|))$ on $I$. Since $|J|_{n} \leq$ $d(J)|\partial J|_{n-1}$ for any interval $J$, we get, for any $(I, \delta)$-fine sequence $\left\{\left(x_{i}, I_{i}\right)\right\}$ with $\sum\left|\partial I_{i}\right|_{n-1} \leq K$,

$$
\sum\left|f\left(x_{i}\right)\right|\left|I_{i}\right|_{n} \leq \sum\left|f\left(x_{i}\right)\right| d\left(I_{i}\right)\left|\partial I_{i}\right|_{n-1} \leq \frac{\varepsilon}{K} \sum\left|\partial I_{i}\right|_{n-1} \leq \varepsilon .
$$

Now comes the definition of our null conditions.

Definition 3.1. Let $I$ be an interval and $F$ be an interval function on $I$.

(i) Given an $n$-null set $E \subseteq I$, we say that $F$ satisfies the condition $\mathcal{N}_{n}$ on $E$ if for any $\varepsilon>0, r>0$ there exists a $\delta: E \rightarrow \mathbb{R}^{+}$such that $\sum\left|F\left(I_{i}\right)\right| \leq \varepsilon$ for any $(E, \delta)$-fine sequence $\left\{\left(x_{i}, I_{i}\right)\right\}$ with $I_{i} \subseteq I$ and $r\left(I_{i}\right) \geq r$ for all $i$.

If $F$ satisfies $\mathcal{N}_{n}$ on every $n$-null set $E \subseteq I$ we say that $F$ satisfies $\mathcal{N}_{n}$ (on $I$ ).

(ii) $F$ is said to satisfy the condition $\mathcal{N}_{n-1}$ (on $I$ ) if for any $\varepsilon>0, K>0$ there exists a $\delta: I \rightarrow \mathbb{R}^{+}$such that $\sum\left|F\left(I_{i}\right)\right| \leq \varepsilon$ for any $(I, \delta)$-fine sequence $\left\{\left(x_{i}, I_{i}\right)\right\}$ with $I_{i} \subseteq I$ for all $i$ and $\sum_{i}\left|\partial I_{i}\right|_{n-1} \leq K$.

R e m ark 3.2. The following facts are obvious (for (ii) use Remark 3.1(i)).

(i) Suppose the $n$-null set $E \subseteq I$ is the disjoint countable union of the sets $E_{i}$. Then $F$ satisfies $\mathcal{N}_{n}$ on $E$ iff $F$ satisfies $\mathcal{N}_{n}$ on all $E_{i}$.

(ii) If $F$ is differentiable at each point of an $n$-null set $E \subseteq I^{\circ}$, then $F$ satisfies $\mathcal{N}_{n}$ on $E$.

LEMma 3.3. Let $f: I \rightarrow \mathbb{R}$ be integrable on the interval $I$ and denote by $F$ the indefinite integral of $f$. Then $F$ satisfies the conditions $\mathcal{N}_{n}$ and $\mathcal{N}_{n-1}$ on $I$.

Pro of. To prove $\mathcal{N}_{n}$ apply Remark 3.1(i) and Lemma 3.1 with parameters $\varepsilon, r, K=1$; to verify $\mathcal{N}_{n-1}$ apply Remark 3.1(ii) and Lemma 3.1 with parameters $\varepsilon, r=1 / 2, K$.

We can now give the following descriptive definition of our integral. 
Theorem 3.1. Let $I$ be an interval and $f: I \rightarrow \mathbb{R}$. Then $f$ is integrable on $I$ iff there exists an additive interval function $F$ on $I$ which is differentiable a.e. on $I$ with $F^{\prime}=f$ and which satisfies the conditions $\mathcal{N}_{n}$ and $\mathcal{N}_{n-1}$ on I. In that case we have $F(J)=\int_{J} f$ for each subinterval $J$ of $I$.

Proof. Assume $F$ to be an additive interval function with the given properties. We will show that $f$ is integrable on $I$ with $\int_{I} f=F(I)$. Denote by $E \subseteq I$ an $n$-null set containing $\partial I$ such that $F^{\prime}=f$ on $I-E$, and let positive numbers $\varepsilon, r, K$ be given.

If $x \in I-E$ choose a $\delta_{1}(x)>0$ such that $\left.\left.|F(J)-f(x)| J\right|_{n}|\leq \varepsilon| J\right|_{n} /\left(5|I|_{n}\right)$ for any subinterval $J$ of $I$ with $x \in J, d(J)<\delta_{1}(x), r(J) \geq r$. Furthermore, since $F$ satisfies $\mathcal{N}_{n}$ on $E$, we choose a $\delta_{1}: E \rightarrow \mathbb{R}^{+}$for the parameters $\varepsilon / 5, r$ and by Remark 3.1(i) we may assume $\sum\left|f\left(x_{i}\right)\right|\left|I_{i}\right|_{n} \leq \varepsilon / 5$ for any $\left(E, \delta_{1}\right)$-fine sequence $\left\{\left(x_{i}, I_{i}\right)\right\}$. According to Remark 3.1(ii) and the fact that $F$ satisfies $\mathcal{N}_{n-1}$ we choose a $\delta_{2}: I \rightarrow \mathbb{R}^{+}$for the parameters $\varepsilon / 5, K$, and we set $\delta=\min \left(\delta_{1}, \delta_{2}\right)$. Let $\Pi=\left\{\left(x_{i}, I_{i}\right)\right\} \in \mathcal{P}(I, r, K, \delta)$. Then

$$
\begin{aligned}
\mid F(I)- & S(f, \Pi, I) \mid \\
\leq & \left.\sum_{\substack{x_{i} \in I-E \\
r\left(I_{i}\right) \geq r}}\left|F\left(I_{i}\right)-f\left(x_{i}\right)\right| I_{i}\right|_{n}\left|+\sum_{\substack{x_{i} \in E \\
r\left(I_{i}\right) \geq r}}\right| F\left(I_{i}\right)\left|+\sum_{r\left(I_{i}\right)<r}\right| F\left(I_{i}\right) \mid \\
& +\sum_{\substack{x_{i} \in I-E \\
r\left(I_{i}\right)<r}}\left|f\left(x_{i}\right)\right|\left|I_{i}\right|_{n}+\sum_{x_{i} \in E}\left|f\left(x_{i}\right)\right|\left|I_{i}\right|_{n} \leq 5 \frac{\varepsilon}{5}=\varepsilon,
\end{aligned}
$$

since $\sum_{r\left(I_{i}\right)<r}\left|\partial I_{i}\right|_{n-1} \leq K$.

The converse follows by Remark 2.2 and Lemmata 3.2-3.3.

Remark 3.3. A null condition of the type $\mathcal{N}_{n}$ was first introduced in [Ju-Kn] to characterize the so called "weak" $n$-dimensional Denjoy-Perron integral (cf. also [Ku-Jar1-3]). Indeed, if we cancel the requirement $\mathcal{N}_{n-1}$ in Theorem 3.1 we are just led to the non-additive $G P$-integral. Thus, it is the additional condition $\mathcal{N}_{n-1}$ which produces an additive integral.

4. Further properties of the integral. In this section we apply Theorem 3.1 to show that our integral extends the Lebesgue integral and to establish a quite general divergence theorem.

4a. Extension of the Lebesgue integral. For any interval $I$ we denote by $\mathcal{L}(I)$ the set of all measurable functions $f: I \rightarrow \mathbb{R}$ for which a finite Lebesgue integral ${ }^{\mathcal{L}} \int_{I}|f|$ exists.

Proposition 4.1. Let $I$ be an interval, and $f: I \rightarrow \mathbb{R}$. Then $f \in \mathcal{L}(I)$ iff $f$ and $|f|$ are integrable, and in that case we have ${ }^{\mathcal{L}} \int_{I} f=\int_{I} f$. 
P r o of. Assume first $f \in \mathcal{L}(I)$ and define an additive interval function by $F(J)=\mathcal{L} \int_{J} f$ for any subinterval $J$ of $I$. According to [Saks], $F$ is differentiable a.e. on $I$ with $F^{\prime}=f$ and it remains to show that $F$ satisfies the conditions $\mathcal{N}_{n}$ and $\mathcal{N}_{n-1}$. Then, by Theorem 3.1, $f$ is integrable on $I$ and ${ }^{\mathcal{L}} \int_{I} f=F(I)=\int_{I} f$. Since $f \in \mathcal{L}(I)$ implies $|f| \in \mathcal{L}(I)$ we will be done.

To see that $F$ satisfies $\mathcal{N}_{n}$ on $I$ let an $n$-null set $E \subseteq I$ and positive numbers $\varepsilon, r$ be given. By the absolute continuity we can find an $\eta>0$ such that $\left|\mathcal{L} \int_{A} f\right| \leq \varepsilon / 2$ for any measurable subset $A$ of $I$ with $|A|_{n}<\eta$. Choose an open set $G \supseteq E$ with $|G|_{n}<\eta$ and for $x \in E$ take a $\delta(x)>0$ such that $B(x, \delta(x)) \subseteq G$. Then for any $(E, \delta)$-fine sequence $\left\{\left(x_{i}, I_{i}\right)\right\}$ with $I_{i} \subseteq I$ we have $\left|\bigcup_{i} I_{i}\right|_{n} \leq|G|_{n}<\eta$ and thus

$$
\sum\left|F\left(I_{i}\right)\right|=\sum_{F\left(I_{i}\right) \geq 0} F\left(I_{i}\right)-\sum_{F\left(I_{i}\right)<0} F\left(I_{i}\right) \leq \varepsilon .
$$

Similarly one checks the condition $\mathcal{N}_{n-1}$ on $I$ (recall that $|J|_{n} \leq d(J)|\partial J|_{n-1}$ for any interval $J$ ).

Conversely, suppose $f$ and $|f|$ to be integrable. Since $f$ is the a.e. derivative of an interval function (by Theorem 3.1), $f$ is measurable according to [Saks]. Thus take $g_{n}=\min (n,|f|) \nearrow|f|$ and observe that

$$
\mathcal{L} \int_{I}|f|=\lim _{n \rightarrow \infty} \mathcal{L} \int_{I} g_{n}=\lim _{n \rightarrow \infty} \int_{I} g_{n} \leq \int_{I}|f|,
$$

by the monotonicity of our integral.

R e m a r k 4.1. We could now formulate the usual convergence theorems for our integral which are reduced via Proposition 4.1 to the classical situation.

4b. The Divergence Theorem with singularities. Let $I$ be an interval, $\vec{v}: I \rightarrow \mathbb{R}^{n}$ be a vector-valued function, and $x \in I$. Then we call $x$ a singularity (of $\vec{v}$ ) if $\vec{v}$ is not continuous at $x$ and if there is an $\alpha$ with $1-n<\alpha \leq 0$ such that

$$
\vec{v}(y)-\vec{v}(x)=O(1)\|y-x\|^{\alpha} \quad(y \rightarrow x, y \neq x, y \in I) .
$$

If $x \in I^{\circ}$ and if $\vec{v}$ is differentiable at $x$ then $\operatorname{div} \vec{v}(x)$ has the usual meaning and at all other points of $I$ we set $\operatorname{div} \vec{v}=0$.

For an interval $I$ we denote by $\vec{n}_{I}$ its usual exterior normal.

LEMMA 4.1. Let $1-n<\alpha \leq 0$. Then there is a positive constant $c(n, \alpha)$, only depending on $n$ and $\alpha$, such that for any interval $J$ and any $x \in J$,

$$
\int_{\partial J}\|y-x\|^{\alpha} d \mathcal{H}(y) \leq c(n, \alpha) d(J)^{\alpha+n-1} .
$$


Proof. For $j \in \mathbb{N}$ let $C_{j}=\left\{z \in \mathbb{R}^{n}: d(J) / 2^{j}<\|z-x\| \leq d(J) / 2^{j-1}\right\}$, and recall that $|B(y, r) \cap \partial J|_{n-1} \leq \beta(n) r^{n-1}$ for any $y \in \mathbb{R}^{n}$ and any $r>0$ with an absolute positive constant $\beta(n)$. Thus

$$
\begin{aligned}
\int_{\partial J}\|y-x\|^{\alpha} & d \mathcal{H}(y) \\
& \leq \sum_{j} \int_{C_{j} \cap \partial J}\|y-x\|^{\alpha} d \mathcal{H}(y) \leq \sum_{j}\left(\frac{d(J)}{2^{j}}\right)^{\alpha}\left|C_{j} \cap \partial J\right|_{n-1} \\
& \leq \beta(n) \sum_{j}\left(\frac{d(J)}{2^{j}}\right)^{\alpha}\left(\frac{d(J)}{2^{j-1}}\right)^{n-1}=c(n, \alpha) d(J)^{\alpha+n-1},
\end{aligned}
$$

with

$$
c(n, \alpha)=\frac{\beta(n) \cdot 2^{n-1}}{2^{\alpha+n-1}-1} .
$$

LEMMA 4.2. Let $I$ be an interval and assume the vector-valued function $\vec{v}: I \rightarrow \mathbb{R}^{n}$ is continuous on $I$ except for an at most countable set $S$ of singularities. Then for any subinterval $J$ of I a finite integral

$$
F(J)=\int_{\partial J} \vec{v} \cdot \vec{n}_{J} d \mathcal{H}
$$

exists and the interval function $F$ thus defined is additive. Furthermore, we have

(i) For every $\varepsilon>0$ there is a $\delta: S \rightarrow \mathbb{R}^{+}$such that $\sum\left|F\left(I_{i}\right)\right| \leq \varepsilon$ for any $(S, \delta)$-fine sequence $\left\{\left(x_{i}, I_{i}\right)\right\}$ with $I_{i} \subseteq I$. In particular, $F$ satisfies $\mathcal{N}_{n}$ on $S$.

(ii) $F$ satisfies $\mathcal{N}_{n-1}$ on $I$.

(iii) F satisfies $\mathcal{N}_{n}$ on $H \cap I$ for any plane $H$.

(iv) If $\vec{v}$ is differentiable at $x \in I^{\circ}$, so is $F$ and $F^{\prime}(x)=\operatorname{div} \vec{v}(x)$.

Proof. In what follows we will often apply the well-known divergence theorem for linear vector-valued functions.

Fix a subinterval $J$ of $I$. Then we can associate with any $x \in \partial J$ a neighborhood $U(x)$ such that either $\vec{v}$ is bounded on $U(x) \cap I$ or $\| \vec{v}(y)-$ $\vec{v}(x)\|\leq K(x)\| y-x \|^{\alpha}$ for $y \neq x, y \in U(x) \cap I$ with a constant $K(x)>0$ and a suitable $\alpha$. Thus $\partial J$ is covered by finitely many neighborhoods $U\left(x_{k}\right)$, and the finiteness of $\int_{U\left(x_{k}\right) \cap \partial J}\left|\vec{v} \cdot \vec{n}_{J}\right| d \mathcal{H}$ follows by Lemma 4.1. The additivity of $F$ is obvious.

(i) Write $S=\left\{s_{1}, s_{2}, \ldots\right\}$ and let $\varepsilon>0$. There are $1-n<\alpha_{k} \leq 0$ and positive numbers $K_{k}$ and $\delta\left(s_{k}\right)$ such that $\left\|\vec{v}(y)-\vec{v}\left(s_{k}\right)\right\| \leq K_{k}\left\|y-s_{k}\right\|^{\alpha_{k}}$ 
for each $y \neq s_{k}, y \in B\left(s_{k}, \delta\left(s_{k}\right)\right) \cap I$. Without loss of generality we may assume $\delta\left(s_{k}\right)^{\alpha_{k}+n-1} \leq \varepsilon /\left(2^{n+k} K_{k} c\left(n, \alpha_{k}\right)\right)$. Let $\left\{\left(x_{i}, I_{i}\right)\right\}$ be an $(S, \delta)$-fine sequence with $I_{i} \subseteq I$. Then in case of $x_{i}=s_{k}$ we have

$$
\begin{aligned}
\left|F\left(I_{i}\right)\right| & =\left|\int_{\partial I_{i}}\left[\vec{v}(y)-\vec{v}\left(s_{k}\right)\right] \cdot \vec{n}_{I_{i}}(y) d \mathcal{H}(y)\right| \\
& \leq K_{k} \int_{\partial I_{i}}\left\|y-s_{k}\right\|^{\alpha_{k}} d \mathcal{H}(y) \\
& \leq K_{k} c\left(n, \alpha_{k}\right) d\left(I_{i}\right)^{\alpha_{k}+n-1} \leq \varepsilon / 2^{n+k} \quad \text { by Lemma 4.1. }
\end{aligned}
$$

Since at most $2^{n}$ of the $x_{i}$ equal a fixed $s_{k}$, we get

$$
\sum\left|F\left(I_{i}\right)\right| \leq \sum_{k} \sum_{x_{i}=s_{k}}\left|F\left(I_{i}\right)\right| \leq \sum_{k} 2^{n} \frac{\varepsilon}{2^{n+k}}=\varepsilon .
$$

(ii) Let $\varepsilon>0, K>0$ be given. For $x \in I-S$ choose a $\delta(x)>0$ such that $\|\vec{v}(y)-\vec{v}(x)\| \leq \varepsilon /(2 K)$ for any $y \in B(x, \delta(x)) \cap I$, by the continuity of $\vec{v}$ at $x$. This defines a function $\delta: I-S \rightarrow \mathbb{R}^{+}$and according to (i) we choose a $\delta: S \rightarrow \mathbb{R}^{+}$for $\varepsilon / 2$. Now let $\left\{\left(x_{i}, I_{i}\right)\right\}$ be any $(I, \delta)$-fine sequence with $I_{i} \subseteq I$ and $\sum\left|\partial I_{i}\right|_{n-1} \leq K$. Then

$$
\begin{aligned}
\sum\left|F\left(I_{i}\right)\right| & =\sum_{x_{i} \notin S}\left|\int_{\partial I_{i}}\left[\vec{v}(y)-\vec{v}\left(x_{i}\right)\right] \cdot \vec{n}_{I_{i}}(y) d \mathcal{H}(y)\right|+\sum_{x_{i} \in S}\left|F\left(I_{i}\right)\right| \\
& \leq \sum_{x_{i} \notin S} \frac{\varepsilon}{2 K}\left|\partial I_{i}\right|_{n-1}+\frac{\varepsilon}{2} \leq \varepsilon .
\end{aligned}
$$

(iii) We may assume $H \cap I \neq \emptyset$, and since $F$ satisfies $\mathcal{N}_{n}$ on $S$ by (i), we only have to show that $F$ satisfies $\mathcal{N}_{n}$ on $(H \cap I)-S$ (cf. Remark 3.2(i)). Let $\varepsilon>0, r>0$ be given. For $x \in(H \cap I)-S$ choose a $\delta(x)>0$ such that

$$
\|\vec{v}(y)-\vec{v}(x)\| \leq \varepsilon^{\prime}
$$

for all $y \in B(x, \delta(x)) \cap I$ with $\varepsilon^{\prime}=\varepsilon r /\left(n 2^{n+1}\left(1+|H \cap I|_{n-1}\right)\right)$ and consider an $((H \cap I)-S, \delta)$-fine sequence $\left\{\left(x_{i}, I_{i}\right)\right\}$ with $I_{i} \subseteq I$ and $r\left(I_{i}\right) \geq r$ for all $i$. Then we get

$$
\begin{aligned}
\sum\left|F\left(I_{i}\right)\right| & =\sum\left|\int_{\partial I_{i}}\left[\vec{v}(y)-\vec{v}\left(x_{i}\right)\right] \cdot \vec{n}_{I_{i}}(y) d \mathcal{H}(y)\right| \leq \sum \varepsilon^{\prime}\left|\partial I_{i}\right|_{n-1} \\
& \leq \frac{2 n \varepsilon^{\prime}}{r} \sum\left|H \cap I_{i}\right|_{n-1} \leq \frac{2 n \varepsilon^{\prime}}{r} 2^{n}|H \cap I|_{n-1} \leq \varepsilon
\end{aligned}
$$

by Lemma 2.1 .

(iv) Assume $\vec{v}$ to be differentiable at $x \in I^{\circ}$, let $\varepsilon>0, r>0$ be given and choose a $\delta(x)>0$ such that $\left\|\vec{v}(y)-\vec{v}(x)-\vec{v}^{\prime}(x)(y-x)\right\| \leq \varepsilon^{\prime}\|y-x\|$ for any $y \in B(x, \delta(x))\left(\subseteq I^{\circ}\right)$ with $\varepsilon^{\prime}=\varepsilon r^{n} /\left(2 n \sqrt{n}^{n}\right)$. Denoting by $J$ any 
subinterval of $I$ with $x \in J, d(J)<\delta(x)$ and $r(J) \geq r$ we see that

$$
\begin{aligned}
\left.|F(J)-\operatorname{div} \vec{v}(x)| J\right|_{n} \mid & =\left|\int_{\partial J}\left[\vec{v}(y)-\vec{v}(x)-\vec{v}^{\prime}(x)(y-x)\right] \cdot \vec{n}_{J}(y) d \mathcal{H}(y)\right| \\
& \leq \varepsilon^{\prime} d(J)|\partial J|_{n-1} \leq 2 n \varepsilon^{\prime} d(J)^{n} \leq 2 n\left(\frac{\sqrt{n}}{r}\right)^{n} \varepsilon^{\prime}|J|_{n} \\
& =\varepsilon|J|_{n},
\end{aligned}
$$

and thus $F$ is differentiable at $x$ with $F^{\prime}(x)=\operatorname{div} \vec{v}(x)$.

THEOREM 4.1 (Divergence Theorem). Suppose an interval $I$ and a vector-valued function $\vec{v}: I \rightarrow \mathbb{R}^{n}$ are given. Assume $\vec{v}$ to be differentiable on $I^{\circ}$ except on at most countably many planes $H_{k}$ and that $\vec{v}$ is continuous on I except for at most countably many singularities. Then a finite integral $\int_{\partial I} \vec{v} \cdot \vec{n}_{I} d \mathcal{H}$ exists, div $\vec{v}$ is integrable on I and

$$
\int_{I} \operatorname{div} \vec{v}=\int_{\partial I} \vec{v} \cdot \vec{n}_{I} d \mathcal{H} .
$$

P r o o f. Using Lemma 4.2 we can define an additive interval function $F$ on $I$ by $F(J)=\int_{\partial J} \vec{v} \cdot \vec{n}_{J} d \mathcal{H}$ for any subinterval $J$ of $I$. Furthermore, $F$ satisfies $\mathcal{N}_{n-1}$ on $I$ and $F$ is differentiable on $I^{\circ}-\bigcup_{k} H_{k}$ with $F^{\prime}=\operatorname{div} \vec{v}$.

Again by Lemma 4.2(iii) and Remark 3.2(i) we see that $F$ satisfies $\mathcal{N}_{n}$ on $T=\partial I \cup \bigcup_{k}\left(H_{k} \cap I\right)$. To see that $F$ satisfies $\mathcal{N}_{n}$ on $I$ take an $n$-null set $E \subseteq I$ and observe that $F$ is differentiable on $E-T$. Hence, by Remark 3.2(ii), $F$ satisfies $\mathcal{N}_{n}$ on $E-T$ and therefore on $E$.

Consequently, by Theorem 3.1, $\operatorname{div} \vec{v}$ is integrable on $I$ and $\int_{I} \operatorname{div} \vec{v}=$ $F(I)=\int_{\partial I} \vec{v} \cdot \vec{n}_{I} d \mathcal{H}$.

Remark 4.2. Note that in Theorem 4.1 the set of those singularities at which $\vec{v}$ is not locally bounded is necessarily finite.

COROLlary 4.1. Let I be an interval and assume the vector field $\vec{v}: I \rightarrow$ $\mathbb{R}^{n}$ is continuous on $I$ and differentiable on $I^{\circ}$. Then $\operatorname{div} \vec{v}$ is integrable on $I$ and $\int_{I} \operatorname{div} \vec{v}=\int_{\partial I} \vec{v} \cdot \vec{n}_{I} d \mathcal{H}$.

\section{References}

[JKS] J. Jarník, J. Kurzweil and S. Schwabik, On Mawhin's approach to multiple nonabsolutely convergent integral, Časopis Pěst. Mat. 108 (1983), 356-380.

[Ju-Kn] W. B. Jurkat and R. W. Knizia, A characterization of multi-dimensional Perron integrals and the fundamental theorem, Canad. J. Math. 43 (1991), $526-539$.

[Ju-No] W. B. Jurkat and D. J. F. Nonnenmacher, A generalized n-dimensional Riemann integral and the Divergence Theorem with singularities, Acta Sci. Math. (Szeged), to appear. 
[Ku-Jar1] J. Kurzweil and J. Jarník, Equivalent definitions of regular generalized Perron integral, Czechoslovak Math. J. 42 (117) (1992), 365-378.

[Ku-Jar2] -, -, Differentiability and integrability in $n$ dimensions with respect to $\alpha$ regular intervals, Results Math. 21 (1992), 138-151.

[Ku-Jar3] - - - Equiintegrability and controlled convergence of Perron-type integrable functions, Real Anal. Exchange 17 (1991-92), 110-139.

[Maw] J. Mawhin, Generalized multiple Perron integrals and the Green-Goursat theorem for differentiable vector fields, Czechoslovak Math. J. 31 (106) (1981), 614-632.

[No] D. J. F. Nonnenmacher, Every $M_{1}$-integrable function is Pfeffer integrable, Czechoslovak Math. J. 43 (118) (1993), 327-330.

[Pf] W. F. Pfeffer, The divergence theorem, Trans. Amer. Math. Soc. 295 (1986), 665-685.

[Saks] S. Saks, Theory of the Integral, Dover, New York, 1964.

ABT. MATHEMATIK II

UNIVERSITÄT ULM

D-89069 ULM, GERMANY

Reçu par la Rédaction le 6.5.1993 